\title{
New concept of vine grape protection - knowledge-based approach \& high tech
}

\author{
B. Gabel
}

NightSky Sro., R\&D Department, Bratislava, Slovakia

\begin{abstract}
Protection of grape vine against pests and pathogens translates into heavy environmental burden more than $100 \mathrm{~kg}$ of toxic chemicals per hectare every year. Limiting factor to ensuring long-term sustainability of plant production are answers to two fundamental questions: WHEN (advisory platform DSS) and WHAT phytosanitary measures must be taken (NDM - zero toxicity concept). DSS: If we want to reduce the number of phytosanitary interventions and reduce toxicological burden in agrocenosis, but simultaneously guarantee rentability of winegrower's work, it is necessary to enter decision-making process of phytosanitary measures with accurate, up-to-date and comprehensive information that are personalized for given vineyard. Advisory platform VITIPORT brings globally applicable protection for viticulture. Its software is based on original algorithms that integrate key abiotic parameters related with pathogens development. They enter mathematical equations in form of coefficients and thus make Vitiport an unique advisory tool built on causal mathematical models. The platform is built on cutting-edge web technologies: SaaS solution using the CLOUD architecture, fast access to data inputs from satellites and terrestrial radar systems. During the whole vegetation period phytosanitary decision-making process is guided by predictions of infection risks for key pathogens for specific "GPS" of the vineyard. End-user information, received a week ahead, is clear, concise, understandable, but most of all personalized. NDM: Methods of plant protection that have been used for decades are based on following formula: plant crop - pathogen - eradication of the pathogen by chemical and/or biological means. NDM concept changes paradigms of plant protection by initiating defense mechanisms of the plant itself instead of using a direct pesticide intervention to fight pathogens. The preparation of NDM substances is a multi-step process employing the most recent knowledge and processes of quantum physics. Diluted concentrate based on natural atomic matrix of plant antibodies is, prior to its use in vineyard, activated with an ultrasonic generator, thereby providing transfer of information about launching plant's defense mechanisms against specific pathogen into water with specific physical parameters. Activated concentrate is finally diluted in the sprayer tank or irrigation system and such final product is applied in the vineyard. Application of NDM substances in agrocenosis does not result in residual contamination of the environment. NDM is therefore a RESIDUE-FREE phytosanitary product, a kind of "vaccine" that activates immune system of the plant.
\end{abstract}

\section{Introduction}

Protection of grape vines against pathogens and pests is, in the classical plant protection concept, based on the use of synthetic or so-called "eco-gently" means. Agro/eco/bio pesticides utilized in grapevine production are a significant and global source of environmental pollution. For this reason, introduction of new methods and means aimed at dramatical reduction of chemicals, respectively their substitution for "toxins free" alternatives, is a necessary and desirable trend in viticulture. All such efforts have to always consider guaranteed yields and acceptable quality of the final product - grape, must, wine.

Protection against fungal pathogens represents $2 / 3$ of the total costs producers must spend on protection during the vegetation season. In years with devastating presence of cryptogamic diseases, European vine-growers have to perform 8-12 fungicidal treatments, in some locations even 17-18 times, only to maintain occurrence of fungal pathogens at an economically viable level. Reflected into environmental burden, this represents more than $100 \mathrm{~kg}$ of toxic chemicals per hectare each year, which we consciously release into agrocenosis.

The backbone of any agricultural production, whether integrated, ecological or organic, must be fully mastered phytosanitary protection. If the aim is to reduce the number of phytosanitary interventions and thus reduce the toxicological burden in agrocenosis, but simultaneously guarantee economic aspects and rentability of winegrower's profession, it is necessary to enter the decision-making process of applying phytosanitary means with accurate, up-to-date and comprehensive information that are personalized for concrete vineyard. Uniform preventive interventions, inaccurate, unaddressed forecasts of phytopathological risks, oftentimes for geographically and climatically different locations within an administrative division of wine-growing regions, are some of the main reasons why objectives fulfillment of the European "anti-pesticide" program (EcoPhyto) was postponed from the original year 2020 to the current 2025. 


\section{Decision support system (DSS)}

Efforts to optimize interventions with fungicides through the mathematical models of prognosis and signalization are evident from multitude of expert literature on various crops. Only early recognition of potential risk of plant infestation by a particular type of pathogen can be an effective prophylactic tool to stop fungal disease at the time of infection risk and animal pests at its key stage - feeding larvae.

\subsection{Cryptogamic pathogens}

Currently, there are several DSS products addressing the issue of signaling protective measures against key cryptogamic pathogens in vineyards. In most cases, these solutions are either built on statistical or multifactorial models.

Predictions of statistical models are based on longterm accurate observations of the occurrence, seasonal dynamics and spatial dispersion of fungal pathogens, combined with identification of key abiotic factors affecting their development. The biological and autecological findings are interconnected with relatively complex meteorological models, where the input data are precise physical parameters from meteorological stations installed in dense topographic networks directly in vineyards. Despite that, these models are not exportable online, as they are based on statistical evaluation of parameters linked to a precisely defined territory and correlated to given biotic and abiotic habitat conditions. Multifactorial models have complicated and overly complex algorithms that evaluate multiple interactions between respective parameters. The pursuit of excessive exactness, evaluation of a number of measurable abiotic factors and their interactions leads to rigidity in decisionmaking process, resulting in vague, unclear and confusing information for practice.

In both cases, final prediction is unclear and complicated. Moreover, both types of models are very sensitive to atypical weather conditions, where the value of prediction is often doubtful in relation to reality in particular field conditions.

\subsection{Tortrix moths}

Signalization of agrochemical interventions against key pest pathogens in European vineyards - tortrix moth caterpillars is based on trapping data of adults into pheromone traps. From these data, a flight curve or in some cases a hatching curve is drafted to determine the optimal time for phytosanitary measure. This method is inadequately inaccurate, since the informative value of the trapping data in relation to the population dynamics of the pest is significantly distorted by several parameters with an unknown accuracy error, e.g. quality of the monitoring system, frequency of control, meteorological factors that affect flight activity of the moths, ... etc.

The inaccuracy of population dynamics monitoring system in vineyards based on pheromone traps is particularly prominent in recent years.

Already at the beginning of pheromone craze on early 70-th last century, Roger Roehrich [4] has pointed out a "remarkable" property of the wild, natural European grape vine moth (EGVM) population. Under natural conditions, they compared the effectiveness of two isomers (E) -7 , (Z) -9 and (E) -7 , (E) -9 dodecadienyl acetate as well as the effectiveness of monoethylene acetate. They found that monoethylene acetate did not appear as an attractant in single experiment, the activity of the (E)-7, (Z) -9 isomer was constant over the two experimental years, while the $(\mathrm{E})-7,(\mathrm{E})-9$ was in one year comparable to that of the $(\mathrm{E})-7,(\mathrm{Z})-9$ isomer, while in the following year it was completely ineffective. In later works by Roehrich [3], attraction differences have been found even between the above-mentioned isomers, not only in individual years, but also between generations within one vegetation season. The cause of this imbalance was genetic heterogeneity of the population in terms of communication signal contained in sex pheromone. What was previously marked by Roehrich [4] as a remarkable feature of wild population is actually normal distribution of occurrence probability of the examined biological property within a population, which is typically expressed by Gaussian distribution curve. Updated identification of EGVM sex pheromone [1] revealed that it is a mixture of three major components (E)-7, (Z)-9 dodecadienyl acetate, (E)-9 dodecadien-1-ol acetate and (Z)-9 dodecenyl acetate with 94:5:1 ratio, furthermore two unsaturated acetates with biological activity during search for mating partner and 5 saturated acetates with unknown biological activity.

Concerns about change of communication signals between the two sexes of target pest have been incorporated during the development of methods for evaluation of sex confusion effect. As the distortion degree of moths' ability to navigate in the agrocenosis, is usually considered to be the reduction in the number of captured moths in pheromone traps or traps with live females. In 1979 stricter criterion for assessing the disruption of the communication system within the natural population was proposed [2]. On the experimental plot, live unfertilized females of tortrix moth were attached to a short nylon fiber and the degree of disruption was determined by the fertilization of these females. This way, inaccuracies in assessing the confusion effect should be eliminated in case that the species also uses other communication signals that have not been yet identified or still unidentified components of the sex pheromone [2] .

Indications of failure of the current sex pheromone formulation in commercial products can be found directly in the official newsletters (BSV - Bulletin de Santé du Végétal), published in France for local winegrowers by the regional agricultural chambers during the vegetation season. For example, the BSV for the region Charente presents a catches of European grapevine moth males from 52 sites into the pheromone traps (Fig. 1). The text states that according to cumulative weekly captures of males, the flight of the first generation peaked between 08-14.05. The average cumulative capture of males during this period was almost 16 individuals (Fig. 1), yet the food traps captured twice as many moths. In the following week (15.-21.05), pheromone traps captured 14 moths on average, the food traps 30 times more, which means $\mathbf{4 2 0}$ moths! The beginning of the first flight was indicated on the same time by both pheromone and food traps, i.e. in the week 24-30.04.2017.

Under normal circumstances, this should be the exact opposite, since the food traps containing a fermenting fluid should be less attractive than selective traps 


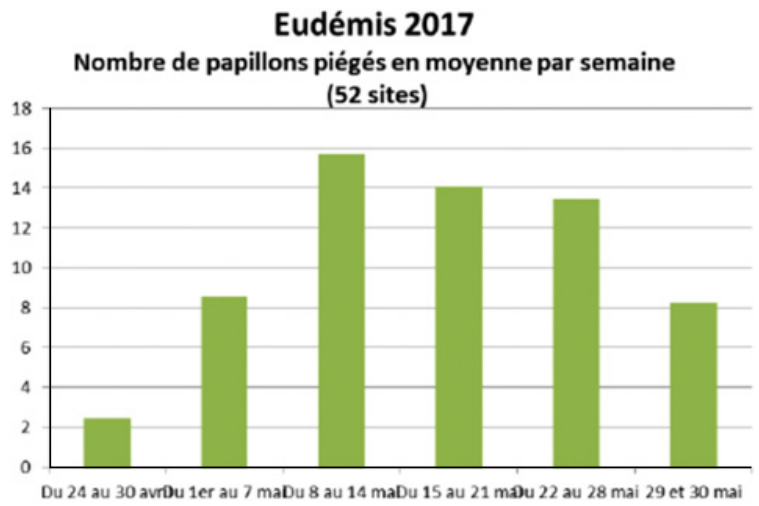

Figure 1. Catches of European grapevine moth males (Lobesia botrana) from the Charente region: Y-axis (average values of weekly catches), $\mathrm{X}$-axis indicates the time period. Source: Bulletin de Santé du Végétal, No.9, 2017.

with sex pheromone. Ratio $14: 420$ is truly shocking. Notwithstanding the fact that in spring, the start of the flight of males and females is always shifted for at least one week due to the reproductive strategy, in entomology known as proterandria. There must be a fundamental error, but it is certain that it is not caused by a change in the bionomy of tortrix moths. The logical and bio-based explanation for the given situation is the low effectiveness of synthetic sex pheromone as a means of communication between the two sexes. If its formulation is failing in pheromone traps which only serve as a monitoring device, there is no reason for excessive optimism also in case of diffusers as means of protection with the method of sexual confusion.

In some countries, breeding cages are used to optimize insecticidal interventions against tortrix, where the wintering pupae of the previous year are exposed in the spring, and the flight and oviposition activity of females on wax paper are observed. Based on these data timing of protective interventions is recommended. Described method is costly, demanding in regard to qualified personnel, inaccurate: diapause pupae spend typically winter in refrigerator, which significantly distorts the process of elimination of diapause in natural conditions, an unrepresentative number of individuals; egg laying on wax paper does not reflect reality, in regard to grapevine phenophases appropriate for oviposition of the natural population and predictive information are geographically limited.

\subsection{Vitiport and IPM}

Advisory platform Vitiport brings a globally functioning solution for grapevine protection. Its concept stems from principles of autecological school, whose foundations were defined in 1952 by experts at the Institute of Experimental Phytopathology and Entomology of the Slovak Academy of Sciences in Ivanka pri Dunaji. Backbone of Ivanka Plant Protection School (IPPS) approach was thorough knowledge of pathogen's life cycle, based on experiments, first in laboratory conditions and subsequently in agrocenosis, the exact determination of abiotic and biotic factors, which determine pest development at population level in monitored agrocenosis. Based on this knowledge, a targeted intervention strategy was proposed at the most sensitive stage of pest development in order to maximize effectiveness of protective measure and minimize the number of phytosanitary measures. Essentially, it was a substitute for conventional chemical methods in plant protection with more eco-friendly, cultural knowledge-based methods that do not endanger human health nor the existence of living nature.

At the turn of the 1960s and 1970s, the so-called Integrated Plant Protection Methods (IPM), began to be promoted in California (UC Berkley, UC Davis), which were fully coherent with principles of IPPS ecological protection. IPM has been declared as a change in thinking, a change in crop protection philosophy, which called for grower's interests to be subordinated to consumer's interests. IPM confirms the opinion that plants suffering from inappropriate agro-technics loose their immunity and significantly activate predator, parasite or pathogen attacking the plant. The focus of IPM is purity of the environment, food hygiene and ecosystem stability. It is an attempt to replace chemical prevention with more natural methods and to correct harmful agents by imitating auto regulatory natural systems. IPM concept has been explicitly defined in 1965 in Rome at the FAO Symposium and Richard Nixon has commissioned the application of the IPM principles to all relevant sectors of the agricultural production of U.S. Environmental Protection Agency (USEPA) in 1972.

Global scalability of advisory platform Vitiport is ensured by its software concept, which is based on algorithms and mathematical models that integrate results of more than 25 years of experiments aimed at determining key life cycle parameters of target pathogens. Critical values of these parameters, obtained from experiments, both in laboratory and natural conditions, enter mathematical equations as coefficients and constants and make Vitiport an unique, globally operating tool, built on causal mathematical models. The platform is built on cutting-edge web technologies using CLOUD architecture and is accessible as SaaS solution (software as a service).

After registration on the website www.vitiport.com, the user identifies location of his vineyard (one click on the map) via Google Maps, and the nearest source of meteorological data in the $1 \mathrm{~km}$ terrestrial grid is found and assigned to the "clicked" vineyard. Starting with the first phenological stages at the beginning of vegetation season until harvest, winegrower's decisionmaking process regarding phytosanitary measures is guided by predictions of infection risks of key pathogens (powdery mildew, downy mildew, grey mold) and predictions of pests (tortrix moth) for specific vineyard, identified through GPS coordinates. End-user receives clear, concise, understandable, but most importantly, personalized information.

Accuracy of prediction algorithms have been validated in different geographic regions (Europe, USA, China, Russia) and since 2017 Vitiport has been tested in number of EU countries: wine-growing regions of France (Saint-Émilion, Cahors, Armagnac, Cognac, Fronton, Medoc, Charentes, Bordeaux, Entre-Deux-Mers), Spain (Rioja), Slovakia (Small Carpathian and South-Slovakian viticultural region), Czech republic (Moravia), Austria (Burgenland) a Italy (Sicily).

Vitiport's prediction models for cryptogamic diseases are based on fulfillment of prerequisite for development of 


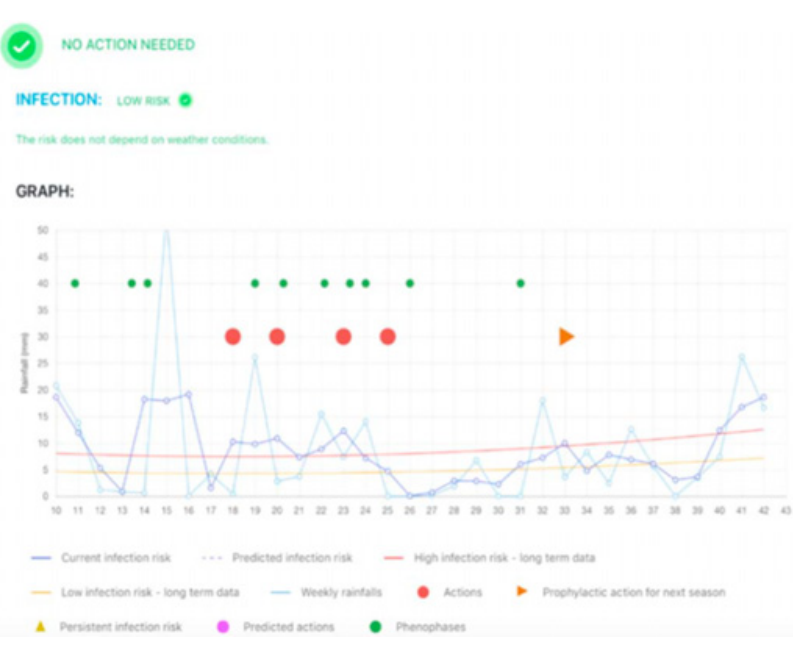

Figure 2. Example of output from online platform Vitiport, module Plasmopara (Downy mildew of grape). Monitored vineyard in region Languedoc-Roussillon (Rodilhan), year 2018.

overwintering stages (primary contamination period) and on fulfillment of meteorological conditions for infection to spread (secondary contamination period). Crucial is calculation of long-term infection curve for given location and curve of current infection risk, which is given by values of 3-week moving rain average. Decision algorithm does not only regard values from these curves, but also time frame with upcoming phenological stages. These are established by combination of two parameters, which determine the growth of grapevine:

a) air temperature (directly affects the speed of enzymatic processes in plant and is expressed in form of $\mathrm{DD}{ }^{\circ} \mathrm{C}$ (degree days). It is summation of differences between average daily temperature and lower threshold temperature for grapevine growth);

b) amount of solar radiation captured by green parts of the plant (energy necessary for photosynthesis in $\mathrm{kWh} / \mathrm{m}^{2}$ ).

Starting with BBCH 09, each Monday winegrower receives predictions of infection risk for specific pathogens (downy mildew, powdery mildew and grey mould) for his vineyard registered on Vitiport with GPS coordinates (Fig. 2). Information about infection risk is valid for current week. Whether there is a need for phytosanitary treatment or not is clearly indicated in regard to pathogen development. Every day, the key parameters are recalculated according to meteorological data from previous day. Last actualisation about the necessity of phytosanitary action is received on Thursday morning. The biggest limit of Vitiport prognosis is reliability of 10-day meteorological prediction. This is not a flaw in algorithms, but an error on the side of predicted meteo data compared to reality. For purposes of Vitiport platform, meteorological data are purchased from authorized state agencies as well as private companies. As we cannot influence the accuracy of meteorological predictions it was necessary to ensure accuracy of Vitiport mathematical models with own solution. Such solution became calculating critical value of weekly rainfall sum in current week, which could have changed an already communicated phytosanitary prediction from the beginning of the week. This value is clearly communicated

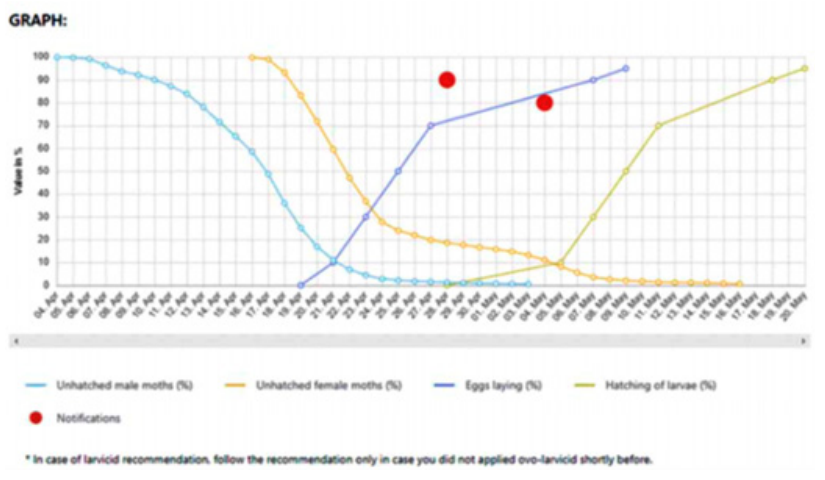

Figure 3. Example of output from online platform Vitiport, module Lobesia (European grape vine moth). Monitored vineyard in region Gironde (Saint-Laurent-d'Arce), year 2018, first generation.

to the end-user in the mobile application. It is updated daily and graphically displayed in form of "loading bar".

Algorithm of tortrix moths development is based on modified function of autocatalytic reactions:

$$
y=100 \cdot\left(1-\frac{A \cdot\left(e^{[(1+A) \cdot B \cdot(x-C)]}-1\right)}{1+A \cdot e^{[(1+A) \cdot B \cdot(x-C)]}}\right)
$$

where $\mathrm{y}=$ percentage of individuals that have not yet completed the developmental stage and $\mathrm{x}=$ sum of effective temperatures.

Upside of this mathematical solution is the fact that individual parameters of the equation are possible to interpret not only mathematically but also biologically. Insect population cannot develop unless the development of the given stage in one individual has been completed. If the development of the others goes on at a high rate, which causes the passing of the whole population into another stage. A logical pre-condition of occurrence of the population in time (mass occurrence of individuals) is the slower developmental rate in the individual than in the population. This pre-condition was fully confirmed when calculated rate coefficients using huge set of experimental data.

We would like to point out a certain connection with the process of chemical autocatalysis. This is based on the fact that the catalyst which considerably speed up the reaction, is a developing inter-product of the noncatalytic reaction which goes on very slowly. If the set of individuals is regarded as a final product, the development of individual can be considere as the first "noncatalytic reaction" and the development of the population as the second "catalytic reaction". In the both reactions the final product is always changed individually. But its rise is conditioned by two processes: (a) change of individual; (b) change of population.

"Chirurgical" accuracy of Vitiport predictions is based on determination of exact values of developmental tresholds for every developmental stage, separately for males and females, and developmental curves based on sums of effective temperatures on population level (Fig. 3). Vitiport's mathematical model assesses also meteorological conditions, which affect induction and elimination of diapause, allowing for precise calculation of onset of first generation and at the end of the season 
the flight of last generation. Vitiport is therefore fully independent of monitoring system based on pheromone traps or oviposition cages stimulating oviposition activity of natural population. It is the only prediction model that takes spring frosts into account and calculates how this factor influences subsequent generations.

Benefits of Vitiport advisory platform can be summarized as following:

1) DSS platform with potential of real-time agronomic solutions on the Northern and Southern Hemisphere, anywhere where there are suitable conditions for grape vine growing;

2) Precise and personalised advisory information, as monitored vineyard is localised through GPS coordinates and input data for prediction models are in $1 \mathrm{~km}$ terrestrial raster;

3) Original prediction models of Vitiport platform ensure, thanks to their causality, relevance of phytosanitary predictions even in years with atypical course of weather. Vitiport therefore becomes a stable advisory tool even for following decades, which, according to all indications of experts, will be influenced by global climatic changes with unpredictable, atypical course of weather in individual years;

4) Evolutive character of portal's architecture creates an opportunity for its horizontal (different crops) as well as vertical use (the same crop, but different pathogens in geographically distant locations);

5) IT solution allows for the most current way of communication with end-user (SMS, mobile and web application, push notifications, email notifications, etc.);

6) It is a product with opportunity of permanent up-date with regard to:

i. new application packages (fertilization plan, phytosanitary treatment plans with regard to antiresistence strategy,..etc.);

ii. new phytopathologic risks (quarantine species of fungal and pest pathogens);

iii. employment of new technologies for viticulture of 3rd Millenium, e.g. spectral imaging and evaluation of vineyard with use of UAV technology.

\section{Plant protection concepts}

Over the past 150 years, countries with intensive agriculture have sought after different practices and means of keeping harmful factors below the threshold of economic harm. On one hand, these efforts shifted us further and generated knowledge, on the other hand, it led to devastating effects on the environment and health of human population.

\subsection{Conventional concept and integrated production}

Crop protection is basically based on active "chemical" fight with a pathogen, where the goal is either its eradication or the effort to control infection risk of the pathogen under the threshold of economic harm. It is a conventional concept of protection classified into the system of so called integrated production. The backbone of this whole process is following scheme: agricultural crop - pathogen - chemical compound dedicated for its eradication.

Accompanying signs of mentioned concept are certain biologic consequences:

1) Emergence of resistency to used chemical substances on pathogen's side. Permanent weakening of plant's health state, i.e. permanent stress due to constant exposure of its vegetative and generative parts to extraneous substances. Man can consider these substances as beneficial, but for the plant itself these are chemicals which it needs to take in, chemically cope with them (phytotoxicity phenomenon!) and build them into its own physiological processes;

2) Presence of residues in agricultural crops and subsequently in foods (bio foods are not exeption!) has dire consequences on health of population;

3) Permanent increase of residual agrochemical compounds in soil and change of physico-chemical properties of the soil (this applies equally to agro or currently popular eco/bio-chemicals!);

4) Subsequent flooding of chemical compounds into deeper soil horizons causes contamination of underground drinking water sources.

Current trend in winegrowing practice is introduction of methods and means aimed at dramatic reduction of synthetic pesticides while guaranteeing yields and longterm multi-level relationships within agrocoenosis. In this case, we are talking about Integrated Production, whose integral part must be constant updating of scientific knowledge from field of plant protection and nutrition, applying such cultivation practices that lead to set goal: reducing the use of pesticides while maintaining qualitative and quantitative crop indicators.

\subsection{Eco/bio concept based on pesticides}

In recent years, chemical substances based on plant and animal extracts (e.g. orange peel, extracts of earthworms, aphids), special blends of inorganic salts inducing an alkaline environment as fungal growth inhibitor (e.g. bicarbonate), or exotic substances based on marine fauna and flora (e.g., seaweed or sea crustaceans) are gaining popularity. For most biopesticides, the effectiveness period is too short, which in practice means frequent repetition of phytosanitary intervention, e.g. protection against tortrix moths with Bacillus thuringiensis (BT) insecticides. With this type of intervention, spraying should be repeated 3 times to achieve the same effectiveness as a single application of an insecticide with ovicidal-larvicidal effect. Of course, a question arises in a wider context, what is actually ecological: $3 \times$ passage, $3 \times$ soil compression, $3 \times$ emission of harmful gases from the tractor, $3 \times$ costs of labour, and $3 \times$ costs of substances? Above all, BT is not a species-specific larvicide, which means it kills the larvae of all species in the cenosis. In the case of fungal pathogens, the effectiveness of these substances is satisfactory in case of low infection risk, but usually fails with moderate and high risk.

Generally speaking, it is the same plant protection concept as the conventional approach, where the grower struggles against the pathogen, but compared to conventional agro chemistry, the $\mathrm{BIO} / \mathrm{ECO}$-effective molecules 
are not sufficiently chemically specified. Therefore, these substances seem to act as a non-residual plant protection solution.

\subsection{New concept of protection - NDM substances}

An ignored possibility of controlling key pathogens in agrocenosis is a complete change in the concept of plant protection, changing the view of the relationship between crop and pathogen. The essence of the NDM concept is not to fight pathogen at the cost of permanent chemical stress for the plant, which is a priori stressed only by the fact that it has to grow in a huge competition of individuals of the same species in the defined area, not to mention other stress factors (nutritional potential of soil, lack/surplus of moisture, sunburn caused by excessive sunlight, a whole range of pathogens - from animal, fungal or plant competitors to weeds). NDM concept changes established paradigms in plant protection: the goal is not a direct intervention to eradicate the pathogen, but to activate plant's defense mechanisms to be prepared in time for the fight with pathogens, with regard to its key phenological phases characterized by different sensitivity, passive or active resistance against a potential attack.

Starting point is an infected plant, attacked by specific and symptomatically clearly defined noxious agent. Plant material from infected vineyard is used for extraction with organic solvents, from which we obtain natural antibodies as plant's response to the attack on its vegetative as well as generative organs.

Extracted antibodies are then chemically specified by atomic matrix and biologically determined through understanding operating mechanism of signals transmitted by pathogens in affected tissues, known as intracellular transducers. These signals activate plant's immune system at multiple levels: production of PR (pathogenesis-related) proteins, enzymes, phytoalexins, production of salicylic and jasmonic acid, ethylene, $\mathrm{pH}$ modification in the cytoplasm, etc. Once the frequencies of pathogen 's signals are specified, we then remake the signals by reconstitution of natural atomic matrix using inorganic components and we store them in specially modified liquid, which acts as transfer medium. Final product - NDM (Natural Defence
Messengers) is a copy of natural atomic matrix that is always specific to each pathogen. NDM substances are messengers of plant antibodies, NDM is therefore a kind of "vaccine" activating plant's immune system, so plant initially sensitive to a pathogen becomes resistant.

Before field application, NDM initial concentrate gets diluted in specific ratio for every type of application (foliar, irrigation, direct application into soil or through biofertilizer with gradual release) and biologically activated with physical ultrasound effect. Application modus of NDM substances may vary among individual winegrowing regions. The correct choice of application method must account for number of factors, especially agronomic specifics of each region, local availability of water sources, spraying technique, and last but not least, local farming practices (e.g. irrigation). This represents application of new technology in viticulture and development of new generation of products with zero residues, with targeted activation of adequate immune pathways of living organism, with zero health risks for workers and zero environmental burdens, including sources of drinking water.

Currently, NDM substances are commercially applied in the EU wine-growing regions. Thanks to wideranging product portfolio the winegrowers have the opportunity to protect their vineyards without any residuals against many cryptogamic pathogens: Powdery mildew of grape, Grapevine downy mildew, Grapevine trunk diseases, Grey mould, Stolbur, Flavescence dorée, Xylella fastidiosa, Grape black rot, Phomopsis cane and leaf spot; but also against frost and herbicide damage: detox after herbicide contamination, regeneration after spring frost, prevention before spring frost.

\section{References}

[1] A. El-Sayed, J. Gödde, P. Witzgall, H. Arn, J. Chem. Ecol. 25, 389 (1999)

[2] S. Rauscher, H. Arn, Ent. Exp. Appl. 25, 16 (1979)

[3] R. Roehrich, Ann. Zool. Écol. anim. 9, 591 (1977)

[4] R. Roehrich, J.P. Carles, Y. Darrioumerle, P. Pargade, B. Lalanne Cassou, Ann. Zool. Écol. Anim. 8, 473 (1976) 